\title{
TEORÍAS DE LA VERDAD Y TEORÍAS DEL SIGNIFICADO: DOS PROGRAMAS ALTERNATIVOS
}

\author{
Álvaro Rodríguez Tirado \\ Ingtituto de linvestigaciones Filóśtíticas \\ Universidad Nagional Autónoma de México
}

1. En su célebre trabajo "Truth and Meaning", publicado hace más de 15 años, Donad Davidson propuso considerar a una teoría de la verdad construida à la Tarski como una teoría del significado para un lengúaje $\mathbf{L}$ dado. De acuerdo a Davidson, una teoría del significado para un cierto lenguaje debe "dar el significado" ${ }^{1}$ de cada una de las oraciones del lenguaje, debe mostrar cómo el significado de una oración es una función del significado de sus partes y de su estructura $y$, por último, debe ser una teoría empirica, o sea, susceptible de verificación mediante la confrontación con los hechos. ${ }^{2}$

Ahora bien, una teoría de la verdad que deba conformarse acorde a la Convención- $V$ descrita por Tarski, ${ }^{3}$ será una teoría que implique, para cada oración o de $\mathrm{L}$, la instanciación del esquema " $\mathrm{x}$ es verdad si y sólo si p", en donde lo que sustituye a " $\mathrm{x}$ " es una descripción estructural de $o$ y lo que sustituye a $p$ es la oración $o$ misma. Existen, no obstante, algunas diferencias importantes entre Tarski y Davidson que obedecen, no a un desacuerdo sustancial entre ambos pensadores, sino a los distintos propósitos que cada uno persigue. Así, para Tarski, la Convención-V representaba un criterio de adecuación de una definición de verdad para un lenguaje dado, mientras que, para Davidson, lo que está en juegoes una teoria de la verdad, o sea, un conjunto de axiomas que implican bicondicionales del tipo descrito, utilizando para estos efectos el concepto: de verdad y otras propiedades semánticas relacionadas con ésta. Por otra parte, en las manos de Tarski, la Convención-V se aplicaba a lenguajes. formales, lenguajes cuyas oraciones expresan lo que expresan independientemente de las circunstancias de emisión; en las de Davidson, en cambio, el predicado "... es verdad" ha de relativizarse a una oración, una persona y un tiempo dados, de manera que, estrictamente, los bicon-

1 Cf. D. Davidson (1967).

2 Ibid.

3 Cf. A. Tarski (1956). 
dicionales aludidos son más bien de la forma de enunciados generales cuantificados universalmente:

(S) ( $t$ ) ( $o$ es verdad pára $S$ en $t$ si y sólo si $p$ ).

Esta segunda diferencia entre la teoría de Tarski y la de Davidson es, en verdad, una cuestión de detalle; más no así la primera. La propuesta de Davidson es subvertir el orden de la explicación: mientras que para Tarski el concepto de verdad puede definirse asumiendo el de traducción, Davidson considera el concepto de verdad como básico o primitivo y pretende extraer de ahí una explicación adecuada de las nociones de traducción e interpretación. ${ }^{4}$ Este cambio en el orden de la explicación incide de manera fundamental en la cuestión ontológica: si nuesaro afán es proveernos de una teoría de la verdad, la ontologia del metalenguaje puede ser exactamente la misma que la del lenguaje objeto. ${ }^{5}$ Además, subvertir la perspectiva tarskiana trae aparejada la obligación de explicar, en forma distinta a como lo hizo Tarski, la verdad de las oraciones de la forma

\section{(V) $S$ es verdad si y sólo si $p$}

o sea, la verdad de los teoremas que implican una teoría de la verdad, es decir, la verdad de las oraciones- $V$ como las llamaremos de aquí en adelante. En la obra de Tarski, las oraciones-V se consideran verdaderas simplemente porque se asume que el lado derecho del bicondicional es una traducción de la oración que se menciona en el lazo izquierdo. Una vez que se ha abandonado este supuesto en la teoría de Davidson, lo que se propone es juzgar la aceptabilidad de las oraciones- $V$ mediante la demostración de que la teoria de la verdad es empiricamente correcta. El desideratum es, pues, que una teoría de la verdad, certificada empíricamente, nos ofrezca interpretaciones de todas y cada una de las oraciones del lenguaje objeto en tal forma que podamos considerar a dichas interpretaciones como su verdadero significado. En resumen, el problema es encontrar restricciones a la teoría que basten para garantizar su uso como una teoría de la interpretación.

Respecto a la obvia conexión que existe entre la definición de verdad que Tarski nos enseñó cómo construir, y el concepto de significado, escribió Davidson:

[La conexión] es ésta: la definición funciona mediante la asignación de condiciones necesarias y suficientes para la verdad de todas

4 Cf. D. Davidson (1973 a), p. 321.

.5 Cf. D. Davidson (1973 b), p. 82. 
las oraciones, y asignar condiciones de verdad es una manera de dar el significado de una oración. Saber el concepto semántico de verdad para un lenguaje es saber en qué consiste el que una oración -cualquier oración- sea verdadera, y esto equivale, en uno de los sentidos que podemos dar a esta frase, a entender el lenguaje. ${ }^{6}$

Ahora bien, si lo que nos dice Davidson está en lo correcto, es claro que su teoría nos muestra cómo es que el significado de una oración depende del significado de sus partes. Dado que existe un número infinito de oraciones- $V$ que deben ser explicadas, la teoría debe proceder en forma recursiva, esto es, la teoría debe mostrar cómo las oraciones-V se derivan de axiomas que asignan propiedades semánticas a los componentes de las oraciones y determinan el resultado semántico de los distintos modos de combinación. Es precisamente en este proceso de derivación de las condiciones de verdad de una oración dada donde puede verse cuál es exactamente la contribución semántica de cada uno de los subcomponentes oracionales. No sería correcto afirmar, entonces, que lo que ha de enseñarnos una teoría de la verdad acerca del significado de las oraciones de un lenguaje podemos encontrarlo en sus teoremas, o sea, en las oraciones- $V$. Las oraciones- $V$ ni siquiera nos dicen algo más acerca de las condiciones en las que una oración en particular es verdadera de lo que ya sabíamos con anterioridad al entender la oración en cuestión. Lo que no debemos perder de vista es, en cambio, que una prueba o demostración de uno de los bicondicionales que exige la Convención-V nos enseña, paso a paso, cómo es que el valor de verdad de una oración depende de la estructura oracional caracterizada recursivamente. Y si dar condiciones de verdad a una oración es dar su significado, entonces, la propuesta de Davidson da un contenido pleno al dictum fregeano de que el significado de una oración es una función del significado de sus partes.

Pero, ¿es realmente cierto que dar el significado de una oración no es más que dar sus condiciones de verdad? Es cierto que a partir de

$$
o \text { significa (en L) que } p
$$

podemos inferir

$$
o \text { es } V(\text { en L) si y sólo si } p \text {; }
$$

pero, lo que Davidson no ha dicho es que el converso también es una

6 Cf. D. Davidson (1967), p. 310. 
inferencia válida - y esto es sencillamente falso. Si asumimos que contamos con la siguiente oración del tipo (1)

(3) o significa (en L) que la nieve es blanca

la inferencia que hemos aceptado — de (2) a partir de (1) - nos permitiría obtener

$o$ es $V$ (en L) si y sólo si la nieve es blanca.

Ahora bien, si leemos al bicondicional que figura en (4) como el bicondicional material, y hacemos uso de la verdad incontrovertible

(5) La nieve es blanca sii el pasto es verde

podemos obtener

(6) o es $V$ sii el pasto es verde.

Y ahora resulta que, si aceptamos que dar el significado de una oración no es más que dar sus condiciones de verdad —esto es, si aceptamos la inferencia de (1) a partir de (2) - tendríamos que aceptar que

$o$ significa que el pasto es verde.

Podría pensarse que el absurdo de esta conclusión se evitaría si entendemos al bicondicional que figura en (2) como un bicondicional estricto, esto es, un tipo de bicondicional que exige, no nada más que las oraciones que lo flanquean tengan el mismo valor de verdad, sino que su coincidencia en estos valores sea necesaria. Desgraciadamente, aún si entendemos la bicondicional de esta manera, es falso que dar el significado de una oración no sea más que dar sus condiciones de verdad (estrictas). Dada la inferencia que hemos aceptado de (2) a partir de (1) y la verdad incontrovertible

(8) La nieve es blanca sii (la nieve es blanca y $2+2=4$ )

obtenemos

(9) $\quad$ es $V$ (en L) sii (la nieve es blanca y $2+2=4$ )

y de ahí podriamos obtener, si la inferencia en cuestión fuese válida, 
(10) o significa (en L) que la nieve es blanca y $2+2=4$

lo cual, como es obvio, es un absurdo.

Nuestra conclusión hasta aquí es que no puede decirse, lisa y llanamente, que "dar las condiciones de verdad de una oración es dar su significado". Lo que muestran el par de ejemplos que hemos ofrecido es que hay, no una, sino, en realidad, un número infinito de teorías de la verdad, que resultan formalmente compatibles con aquella que, intuitivamente, consideramos la correcta. Pero, cacaso podríamos pensar que un hecho tan elemental, como sin lugar a dudas es éste, le pasó por alto al Davidson que escribió "Truth and Meaning"?

2. John Foster criticó el programa de Davidson en su artículo "Meaning and Truth Theory" " siguiendo, fundamentalmente, la línea de argumentación que he recogido arriba. Veamos el argumento de Foster. Consideremos un lenguaje $\mathrm{L}$, por demás sencillo, que consta de un solo predicado diádico $P$, la barra (stroke) como único conectivo veritativofuncional con su significado normal, el cuantificador universal ' $\wedge$ ' con su significado normal y un conjunto denumerable de variables ordenadas como en la siguiente lista

$$
v_{1}, v_{2}, v_{3}, \ldots
$$

los paréntesis '(', ')' $\mathrm{y}$, por último, asumimos que todas las oraciones de $\mathrm{L}$, abiertas o cerradas, se construyen a partir de estos símbolos primitivos siguiendo las reglas estándard de formación del cálculo de predicados.

El primer paso en la construcción de una teoría de la verdad para $L$ consiste en asumir el concepto de secuencia infinita de objetos, el cual, en realidad, debe entenderse como una asignación, o sea, como una función, ya que cada secuencia asigna un valor único a cada variable de $\mathbf{L}$, a saber, el valor $v_{n}$ de la secuencia $\Sigma$ es sencillamente el n-ésimo elemento de $\Sigma$. El segundo paso estriba en encontrar una traducción al español del predicado $P$, la cual, en el ejemplo de Foster, nos la da el predicado "parte de". Llegamos por fin al núcleo mismo de la teoría de la verdad para $\mathbf{L}$. Este consiste en una caracterización recursiva de la relación de satisfacción entre asignaciones y oraciones:

i) $\Sigma$ satisface una oración atómica de $\mathrm{L}$-es decir una fórmula que consiste de $P$ y un par de variables - sii el objeto que $\Sigma$ asigna a la primer variable es parte del objeto que $\Sigma$ asigna a la segunda variable;

7 John Foster (1976). 
ii) $\Sigma$ satisface a una oración de la forma $A \frown$ barra $-B$ sii $\Sigma$ no satisface a A o B;

iii) $\Sigma$ satisface una oración de la forma ' $\wedge$ ' $\nabla_{n}-A$ sii $A$ es satisfecha por cualquier asignación que, si difiere de $\Sigma$, difiere de ella en, a lo más, el n-ésimo lugar.

Si añadimos a estas cláusulas los axiomas relativos a la teoría de las secuencias, tendríamos una teoría de la verdad para $L$, o sea, una teoría $\Theta$ como la llamaré de aquí en adelante: para cada oración cerrada $o$ en $L, \Theta$ nos ofrece una instanciación del esquema " $x$ es verdadera sii $p$ " si sustituimos una descripción estructural de $o$ por $x$ y una traducción de $o$ por $p$.

Pero, si bien $\Theta$ nos da una caracterización adecuada de verdad-en-L, no nos da, en palabras de Foster, "ni siquiera de una manera oblicua o indirecta, una caracterización del significado". ${ }^{8}$ La razón es, de nuevo en palabras de Foster, "que para toda oración- $L$ hay un número infinito de interpretaciones relevantemente distintas consistentes con $\Theta " .8$ En el caso del lenguaje $L$ que venimos considerando ahora, la cláusula crucial es la relativa al predicado $P$. Todo lo que nos dice esta cláusula es que, para cualesquiera dos enteros, $i$ y $j$, una asignación satisface $P \frown \mathbf{v}_{\mathbf{1}} \frown \mathbf{v}_{j}$ sii el i-ésimo es una parte de j-ésimo elemento. Pero, decir que $P$ se aplica a todos los pares ordenados de objetos que guardan entre sí la relación de parte-todo y sólo a ellos, es perfectamente compatible con cualquier otra interpretación de $P$ que coincida en su extensión. Así, si sustituimos en la cláusula $(i)$ la designación del predicado $P$ por la designación de un predicado $P^{\prime}$ que sea co-extensivo al primero, no alteramos en lo absoluto su verdad. Por tanto, si encontramos un predicado $P^{\prime}$ co-extensivo con $P$, pero que difiera de éste en cuanto a su sentido, no hay nada en ninguna de las cláusulas de $\Theta$ que nos impida interpretar a $P$ como si significase lo que $P^{\prime}$ significa - sea esto lo que fuere. $Y$ esto parecería contradecir abiertamente lo que Davidson afirmó al escribir: "He argumentado en otros trabajos que una teoría de la verdad, modelada de acuerdo a una definición de verdad del tipo de Tarski, nos dice todo lo que debemos saber acerca del sentido." 10

Nada hay más sencillo que encontrar un predicado $P^{\prime}$ co-extensivo con $P$, pero que difiera en cuanto a su sentido: Tómese cualquier oración que resulte verdadera en español, digamos, "el pasto es verde". Pensemos ahora que el predicado $P^{\prime}$ podría ser traducido correctamente así "el pasto es verde y ... es parte de ...". Dado que el pasto es verde,

8 Ibid.

9 Ibid.

10 D. Davidson (1979), p. 9. 
$P$ y $P^{\prime}$ son co-extensivos: no hay un sólo par de objetos al que uno se aplique y no el otro, pero es obvio que su significado es distinto.

La moraleja es clara: si bien es cierto que la teoría $\Theta$ fija la extensión del predicado $P$, no hace nada por fijar su sentido. Un argumento similar al que hemos expuesto podría darse en relación a la manera de entender el conectivo veritativo-funcional y el cuantificador universal. Por esta razón, la conclusión de Foster parecería ser obvia: sean cuales fueren los méritos de $\Theta$ como una teoría de la verdad, $\Theta$ es una pésima teoría del significado. ${ }^{11}$

3. La crítica de Foster, y los argumentos que la inspiran - sucintamente expuestos en la sección anterior- ignoran que Davidson ha estado siempre consciente de que, en donde Tarski asumió la noción de traducción, habría que buscar las restricciones empíricas que viniesen a certificar a una teoría de la verdad como una teoría de la interpretación. En su réplica a Foster, Davidson escribio:

Que deberían añadirse restricciones emplricas a las restricciones formales si teorías aceptables de la verdad han de incluir únicamente aquéllas que sirvan para la interpretación, lo veía con claridad incluso desde que escribí "Truth and Meaning". ${ }^{12}$

En efecto, ya en el artículo de 1967, Davidson enfatizaba el carácter empírico de la teoría propuesta:

Una teoría del significado... es una teoría empírica, y su ambición es explicar el funcionamiento de un lenguaje natural. Como cualquier otra teoría, puede verificarse al comparar algunas de sus consecuencias con los hechos. ${ }^{13}$

Cuando el lenguaje para el cual damos una teoría de la verdad está contenido (es parte de) el lenguaje de la teoría, la verificación de la teoría es cosa por demás sencilla, por no decir trivial. Pero no así en el caso en que intentamos dar una teoría de la verdad en nuestro lenguaje, digamos, en español, de otro lenguaje distinto. Aquí, el intérprete intentará ofrecer un apareo de oraciones que el nativo toma como verdaderas (o falsas) con oraciones que el mismo intérprete toma como verdaderas (o falsas); pero se debe mostrar cómo es esto posible sin asumir que el intérprete tenga, o haya tenido, un acceso directo a las equivalencias entre el lenguaje del nativo y el suyo propio. Esto se logra si asumimos que los nativos asienten, en su mayor parte, a oraciones verdaderas y

11 J. Foster, op. cit.

12 Op. cit., p. 35.

13 En la p. 311. 
disienten, en su mayor parte, a oraciones falsas. Este es el mallamado "Principio de Caridad": no se trata de un cierto altruismo por parte del intérprete, algo de lo cual podría fácilmente prescindir. Asumir que las aseveraciones del nativo se dan en un contexto en el cual la mayoría de sus creencias son verdaderas es algo inevitable si es que la comunicación ha de proceder tarde o temprano, e inevitable también para detectar el error en sus afirmaciones, cuando de error se trate. El intérprete debe, por tanto, maximizar el acuerdo con el nativo. Cualquier otra alternativa es ininteligible: aceptar demasiada discordancia no es tan sólo aceptar un error multiplicado; es socavar la base misma sobre la cual puede hablarse de error (o de cualquier otra cosa) en lo absoluto.

Sería falso pensar, entonces, que Davidson pasó por alto las restricciones empíricas que han de añadirse a los requisitos formales que una buena teoria de la verdad (para un lenguaje $L$ ) debe satisfacer para poder fungir como una teoría de la interpretación. $\mathrm{Y}$ esto es así, como ya hemos visto, desde el momento mismo en que escribió "Truth and Meaning". No cualquier teoría que pueda darnos, correctamente, las condiciones de verdad de toda oración de un lenguaje, puede servir como una teorfa de la interpretación, puede ser considerada como una teoria del significado. Ahora bien, es cierto que, según Davidson, la certificación empírica de una teoría de la interpretación debe apelar a un tipo de evidencia que no haga uso de nociones tales como significado, sinonimia, interpretación, etc. ${ }^{14}$ Pero es de suma importancia notar que Davidson jamás ha pensado que sea posible explicar el funcionamiento de un lenguaje, y la noción misma de significado, sin apelar a nociones intensionales. Los conceptos de creencia e intención figuran, como veremos más adelante, en forma esencial en las explicaciones davidsonianas, y el mismo Davidson ha dejado fuera de toda disputa su creencia de que es imposible intentar reducir dichos conceptos a otras nociones consideradas más cientificas o que se encuentren más enraizadas en la conducta:

Lo que he intentado hacer es dar una explicación del significado (interpretación) sin hacer un uso esencial de conceptos lingüísticos aún sin explicar. Ningún plan mío se vería arruinado [como pensaba Foster] si para decir lo que un intérprete sabe, es necesario usar una de las llamadas nociones intensionales - noción que armonice con la creencia, intención, etc. ${ }^{15}$

En su articulo "Belief and the Basis of Meaning", Davidson fue aún más explícito:

14 Cf. D. Davidson (1973 a).

15 Cf. D. Davidson (1976), p. 38. 
Las teorías del significado y la creencia no requieren de ningún objeto exótico, pero sí utilizan conceptos que las ponen en una categoría distinta a la de las ciencias físicas y otras ciencias no psicológicas: los conceptos de significado y creencia no son, de una manera muy básica, reducibles a conceptos físicos, neurológicos e incluso conductuales... Son más bien los métodos que tenemos que invocar al construir teorías del signifificado y la creencia lo que nos asegura de la irreductibilidad de esos conceptos esenciales a dichas teorías. Cada interpretación y atribución de una actitud es un paso dentro de una teoría holista, una teoría necesariamente gobernada por el interés, por la consistencia y la coherencia general con la verdad, y es esto lo que separa de por vida a estas teorias de aquéllas que describen objetos carentes de mente o describen a objetos como si careciesen de ella. ${ }^{18}$

Antes de entrar a elucidar el problema de lo que verdaderamente sabe el intérprete, veamos con mayor detenimiento la evidencia de que dispone para construir su teoría de la interpretación. Es claro que en los primeros estadios de la interpretación radical, el intérprete no puede apelar a las creencias e intenciones de los hablantes, ya que saber, detalladamente, cuáles son éstas, presupone haber entendido las emisiones a que los hablantes han dado lugar:

La dificultad central es que no podemos tener la esperanza de adjudicar un sentido a la atribución de intenciones finamente discriminadas independientemente de la interpretación del discurso. ${ }^{17}$

La interdependencia de la creencia y el significado hace imposible considerar a las creencias y las intenciones como evidencia de que las oraciones- $V$ son verdaderas. Un hablante considera a una oración como verdadera en función de (i) lo que esa oración significa y (ii) lo que el sujeto cree. Saber que una persona considera a una oración dada como verdadera, y saber también lo que esa oración significa, nos permite inferir su creencia; si tenemos información suficiente acerca de sus creencias, podemos inferir el significado de sus oraciones. Pero la interpretación radical debe descansar en evidencia que no asuma ni el conocimiento de los significados de las oraciones, ni el conocimiento detallado de las creencias.

Davidson propone considerar la actitud de aceptar una oración como verdadera, de tomarla como verdadera, como el punto de partida. Esta es, en efecto, una actitud proposicional, una creencia, ni más ni menos.

16 Cf. D. Davidson (1974), p. 322.

17 Cf. D. Davidson (1973 a), p. 315. 
El valor de esta noción frente a otras es que se trata de una actitud que podemos observar ante cualquier oración y no exije, por tanto, que seamos capaces de discriminar finamente entre los contenidos de las creencias:

Es plausible afirmar que podemos decir cuándo un hablante considera a una oración como verdadera sin que sepamos lo que quiere decir con esa oración, o qué creencias contempla acerca del desconocido tema de que trata o qué intenciones detalladas lo llevan a emitirla. ${ }^{18}$

Tenemos, entonces, por una parte, oraciones-V de la forma

(V) "It is raining" es verdad-en-inglés cuando $x$ la emite en el tiempo $t$ sii está lloviendo cerca de $x$ en $t$,

y, por la otra, un tipo de evidencia (E) como la descrita a continuación:

(E) Donald pertenece a la comunidad lingüistica inglesa y Donald considera que "It is raining" es verdad el sábado por la tarde, y está lloviendo alrededor de Donald ese sábado por la tarde.

Pero es obvio que Donald puede ser víctima de una alucinación y creer que está lloviendo a su alrededor cuando en realidad no lo está. Sucede entonces que (E) debe considerarse como evidencia, pero no como una evidencia de tipo concluyente. Claro que Donald, o cualquier otra persona, puede equivocarse. Todo lo que se requiere por parte del intérprete es que intente maximizar el acuerdo — no se le adscribe la imposible tarea de hacer de los nativos unos seres omniscientes.

Ahora bien, es cierto que lo que eventualmente queremos verificar son las oraciones-V. Pero no consideradas aisladamente:

La esperanza es que al imponer restricciones formales y empíricas a la teoria como un todo, las oraciones- $V$ individuales servirán en efecto para darnos interpretaciones. ${ }^{19}$

El criterio es pues que la totalidad de las oraciones-V se adecúen en forma óptima a las oraciones que los hablantes nativos consideren verdaderas. Es así como llegamos a obtener el efecto deseado: 
...extraer un concepto rico (aquí algo razonablemente cercano a la traducción) a partir de finos trocitos de evidencia (aquí los valores de verdad de las oraciones) imponiendo una estructura formal a un buen número de estos trozos. ${ }^{20}$

Nótese que verificar si una teoría de la verdad es empíricamente correcta, trac consigo tan soló objervar la conducta y ia actitud de los habilantes respecto de las oraciones. Obviamente, el hecho de que dicha teoría satisfaga la Convención-V significa que las oraciones son analizadas como construcciones a partir de componentes suboracionales, términos singulares, predicados, cuantificadores, conectivos oracionales, y que el vínculo de estas expresiones con entidades extralingüísticas se lleva a cabo por medio de la caracterización de la noción de satisfacción. Pero todo esto debe considerarse nada más como construcciones teóricas, como la herramienta de una teoría empírica del significado, cuya verdad deberá confirmarse mediante el éxito en sus predicciones de las condiciones de verdad de las oraciones. ${ }^{21}$

$¿ Y$, qué decir de la tesis quineana de la indeterminación de la traducción? En diversos trabajos, Davidson ha llamado nuestra atención al hecho de que, una vez reunida toda la evidencia, seguiria habiendo toda una amplia gama de teorías de la verdad que se adecúen a la evidencia de igual manera: no habría, por tanto, la teoría de la verdad o la teoría de la interpretación. Empero, Davidson parecería haber cambiado su opinión respecto a la importancia de aceptar dicha indeterminación semántica. En "Semantics for Natural Languages", Davidson escribió:

Pienso que Quine está en lo correcto al sostener que un grado importante de indeterminación prevalecerá después de haber reunido toda la evidencia; un buen número de teorías de la verdad significativamente distintas se adecuarán a la evidencia de igual manera. ${ }^{22}$

Pero, en "Radical Interpretation" sostuvo:

Pero la indeterminación resultante no puede ser tan grande como para que ninguna teoría que cumpla los requisitos [formales y empíricos] pueda servir para la interpretación. ${ }^{23}$

$\mathrm{Y}$, en forma más categórica, en su artículo "Belief and the Basis of Meaning", escribió

20 Cf. D. Davidson (1973 b), p. 84 .

21 Ibid., p. 84.

22 Cf. D. Davidson (1970), p. 22.

23 En la p. 327. 
La indeterminación del significado o de la traducción no representa el haber fallado en la captura de distinciones importantes; enfatiza el hecho que ciertas distinciones aparentes no son importantes. ${ }^{24}$

Parecería entonces que, si bien existe indeterminación del significado (y de la traducción) en el sentido de que siempre habrá un buen número de teorías (manuales) alternativas que se adecúan a la evidencia empirica, este hecho no es importante. Davidson explota una analogía con la teoría de la decisión para mostrar lo inocuo de dicha indeterminación: "si los números (1), (2) y (3) captan las relaciones relevantes en el valor subjetivo de tres alternativas, entonces, los números $(-17),(-2)$ y $(+13)$ lo harian también. La indeterminación de este tipo no puede representar una preocupación genuina". ${ }^{25}$

4. Hemos visto ya (§3) que el que un hablante considere a una oración como verdadera es una función de, por una parte, lo que el hablante quiere decir mediante la emisión de la oración en cuestión y, por la otra, lo que dicha persona cree. Por esta razón y, dado que nuestro punto de partida en nuestra labor interpretativa es simplemente la emisión honesta, por parte del hablante, de una oración dada, no podemos inferir el significado de dicha oración sin saber su creencia, y no podemos inferir lo que cree sin saber cuál es el significado de la oración en cuestión.

A partir de estas consideraciones, aunadas al hecho de que no es posible fundar una teoría del significado en un descubrimiento previo de las creencias e intenciones de los hablantes, Davidson concluye que

al interpretar emisiones partiendo de cero —en la interpetación radical - debemos de alguna u otra forma ofrecer, simultáneamente una teoria de la creencia y una teoría del significado. ${ }^{26}$

El problema es cómo es esto posible. Nuestra evidencia, a partir de la cual debemos inferir las creencias y el significado, es esa actitud de considerar a una oración como verdadera (holding true) de la cual hemos venido hablando. En otras palabras, asumimos que sabemos cuándo un hablante considera a una oración como verdadera y queremos averiguar el significado de la oración y las creencias del hablante. Si tuviésemos ya en nuestras manos la interpretación correcta de sus palabras, podríamos inferir sus creencias_e intenciones; si supiésemos lo suficiente de sus creencias e intenciones, averiguariamos sin dificultad alguna el signifi-

24 En la p. 322.

25 Ibid.

26 Ibid., p. 312. 
cado de sus palabras; pero, ¿cómo saber cuáles son sus creencias e intenciones sin una previa teoría de la interpretación?

Existe una analogía - -en realidad existe algo más que una analogía como veremos después- que Davidson ha explotado con la teoría de la decisión. En los juegos de azar, nuestra elección es una función de dos factores: (i) los valores que asignamos a cada uno de los resultados y (ii) la probabilidad que asignamos a esos resultados condicionada a nuestra elección. Nos encontramos nuevamente con lo que parecería ser un círculo inquebrantable:

Dadas las creencias (i. e., las probabilidades subjetivas) del agente sería fácil computar sus valores relativos a partir de sus elecciones; dados sus valores, podemos inferir sus creencias. Pero si sólo tenemos sus elecciones ¿cómo podríamos averiguar tanto sus creencias como sus valores? ${ }^{27}$

Es a F. P. Ramsey a quien debemos la sugerencia de una manera de romper el círculo. La idea de Ramsey estribó en mostrarnos cómo encontrar un suceso cuya ocurrencia la considera el agente tan probable como su no-ocurrencia, sobre la base exclusivamente de sus elecciones: si ante dos apuestas - la primera consistente en que si ocurre $\mathrm{E}$ el agente se lleva $\$ 1000.00$ y si no ocurre no se lleva nada, y la segunda consistente en que si $\mathrm{E}$ ocurre el agente no se lleva nada y si no ocurre se lleva $\$ 1000.00$ - el agente es indiferente, quedaría mostrado que el agente juzga tan probable que ocurra $\mathbf{E}$ como que no ocurra.

Esto resuelve, para la teoría de la decisión, el problema de cómo separar la probabilidad subjetiva de la utilidad subjetiva ya que, una vez que descubrimos un suceso como $\mathbf{E}$, es posible jerarquizar otros valores y entonces determinar las probabilidades subjetivas de todos los valores. ${ }^{28}$

Davidson observa una interdependencia notable entre la teoría de la decisión y la teoría de la interpretación. No se trata tan solo de una analogía, como ya lo había anticipado. El estudio de la teoría de la decisión nos muestra algo mucho más profundo que un simple paralelo entre el problema del significado y las creencias, por una parte, y el problema de las probabilidades subjetivas y los valores asignadas, por la otra. La interdependencia entre ambas teorías puede verse claramente si nos preguntamos: ¿cómo sabe el que lleva a cabo el experimento cuáles son las preferencias del agente? Obviamente, el agente tendrá que describir la

27 Ibid., p. 313.

28 Ibid., p. 314. 
alternativa que ha seleccionado. $\mathrm{Y}$ ¿cómo sabe el oyente lo que significan esas palabras? Es necesario apelar a una teoría de la interpretación. Hay, por otra parte, un dificultad obvia para decidir cuándo una persona acepta una oración como verdadera, y es aquí donde la teoría de la decisión puede brindar cierta ayuda a la teoría de la interpretación. La teoría de la decisión nos permite ver con toda claridad a las creencias en su papel explicativo al racionalizar las preferencias o elecciones de un agente. Las preferencias de un agente se muestran en sus elecciones - tal y como el asentimiento a una oración se muestra en la aserciónpor lo que parecería recomendable sustituir la creencia de que una oración es verdadera por otra noción, la de preferencia entre oraciones - la preferencia de que una oración, en lugar de otra, sea verdadera. ${ }^{29}$

El camino parecería estar despejado para la elaboración de una teoría unificada de la decisión y de la interpretación. Por lo que toca al apoyo evidencial que puede darse a una teoría de la interpretación, la teoría de la decisión ha ofrecido un apoyo sustancial. Recordemos que el punto de apoyo evidencial lo constituye la actitud de los hablantes de una comunidad al considerar a una oración como verdadera. Pero como escribe Davidson

es obvio que una interpretación correcta de las palabras de un hablante dependerá, en muy buena medida, de nuestro conocimiento del grado en el que un hablante considera que la verdad de una oración apoye la verdad de otra. ${ }^{30}$

Por tanto, lo que se requiere en una teoría de la interpretación no es solo el conocimiento de lo que causa que un hablante considere a una oración como verdadera, sino el grado de su creencia en esta verdad. Es esto lo que hace que Davidson concluya que

La teoría del significado... y la teoría bayesiana de la decisión están evidentemente hechas la una para la otra. La teorfa de la decisión debe liberarse del supuesto de que existe ya un conocimiento determinado independientemente del significado; la teoría del significado exije una teoría del grado de creencia para poder asi hacer un uso serio de las relaciones de apoyo evidencial. ${ }^{31}$

Una teoría unificada deberá, por tanto, darnos a la vez el grado de creencia, los valores relativos y una interpretación del discurso sin asumir ninguno de ellos:

20 Ibid., p. 316.

30 D. Davidson, "Towards a Unified Theory of Meaning and Action" (en prensa). 31 Ibid., p. 8. 
Lo que se requiere para una teoría unificada... es alguna actitud proposicional simple, la cual pueda adscribirse plausiblemente a un agente sin poseer un conocimiento detallado de sus creencias, preferencias o significados lingüísticos y a partir de la cual se pueda extraer una teoría de los grados de creencia, comparaciones entre los grados de deseo y un método para interpretar sus oraciones. ${ }^{32}$

La actitud que Davidson recomienda como básica es: el agente prefiere como verdadera a una oración determinada y no a otra. El intérprete parte entonces de este tipo de información, del hecho de que los hablantes prefieren unas oraciones como verdaderas y no otras. Si a partir de este tipo de evidencia podemos inferir el grado de creencia en oraciones tendremos una teoría unificada, toda vez que ya se ha mostrado cómo, a partir del conocimiento del grado en que una persona considera a una oración como verdadera, se puede construir una teoría del significado y la creencia.

En este punto, Davidson apela a la formulación de la teoría de la decisión que unifica a los objetos de preferencia, los objetos a los cuales se asignan probabilidades subjetivas, y los objetos a los cuales se asignan valores relativos, considerándolos a todos como proposiciones. Dicha formulación se debe a $\mathbf{R}$. Jeffrey. Jeffrey mostró cómo extraer las probabilidades subjetivas y los valores a partir de preferencias para considerar ciertas proposiciones como verdaderas. Pero Davidson no puede asumir que entendemos las proposiciones sin incurrir en una petición de principio, por lo que nos propone considerar a las oraciones y hacer depender toda esta empresa en que se puedan identificar las conectivas veritativo-funcionales:

Si comenzamos con oraciones en lugar de proposiciones, entonces nuestro problema quedaría resuelto si tan solo pudiésemos identificar a las conectivas veritativo-funcionales. Identificadas éstas, Jeffrey ha mostrado cómo pueden fijarse las probabilidades y la deseabilidad subjetiva [subjetive desirability] de todas las oraciones en el grado deseado; y he argumentado que esto basta para dar una teoría de la interpretación de las oraciones. ${ }^{33}$

Davidson confiesa no estar completamente seguro de que pueda hacerse tal cosa. No obstante, "parece" —nos dice Davidson- "probable que sí pueda hacerse". Por ejemplo, si descubriésemos un operador $O$ de oraciones tal que la aplicación del operador a todo par de oraciones invierta el orden de la preferencia, $i$. e., si es el caso que, si se prefiere $o$ a $o^{\prime}$, 
entonces, se preferiría $O o^{\prime}$ a $O o$, entonces, tal operador tenderá a ser entendido como la negación. Hay algunos problemas técnicos que parecerían ser de detalle. Si los hacemos a un lado, podríamos apelar al mismo tipo de evidencia que nos sirvió para detectar la negación y descubrir otro operador de oraciones tal que, cuando se aplica a un par de oraciones, el resultado es verdadero si y sólo si ninguna de las operaciones componentes lo es. $\mathrm{Y}$ como es de todos sabido, si tenemos este operador, tenemos también el resto de los conectivos.

5. Las secciones anteriores no han tenido ninguna otra pretensión que la de presentar un resumen de la posición adoptada por Davidson ante la pregunta "¿qué es una teoría del significado?" Dentro de los muchos problemas que aún quedan por tratar me interesan, particularmente, la cuestión de qué tipo de conocimiento tienen los hablantes de un lenguaje de la teoría del significado propuesta, la compatibilidad o incompatibilidad de tales teorfas con los argumentos de Wittgenstein sobre lo que es seguir una regla, y la disputa realismo vs. antirrealismo en semántica. No voy, sin embargo, a intentar desarrollar ninguno de estos temas aquí. Mi interés por describir el "programa davidsoniano" con cierto detalle res. ponde a la necesidad de tener un marco de discusión para la evaluación de teorias alternativas del significado y la discusión de problemas filosóficos relacionados como los que acabo de mencionar. El haber escogido para estos efectos a la teoría de Davidson, no se debe a un prejuicio en favor de esa teorfa, sino al hecho de que, hoy por hoy, no hay ningún otro escenario que supere al que Davidson nos ha propuesto para el planteamiento de problemas que preocupan al filósofo del lenguaje. $\mathrm{Ha}$ 'cer de la explicación sistemática de la verdad la tarea central de la semántica empírica es tan solo, como algún día dijera Dadivson, enunciar en una forma más clara nuestros viejos propósitos.

Este es un supuesto compartido por una abrumadora mayoria de quienes se encuentran trabajando en esta área de la filosofía, al menos dentro del mundo anglosajón. Pero también en el mundo hispano-hablante hay quienes comparten el supuesto, y fue precisamente la publicación de un articulo titulado "Significado, Verdad y Comprensión", ${ }^{34}$ que me estimuló, al menos parcialmente, a llevar a cabo este trabajo. Los autores del citado artículo, García Suárez y Valdés Villanueva - a quienes de aquí en adelante me referiré así: 'GS-VV'- discuten la propuesta del filósofo inglés John McDowell,"35 intentando mostrar que "...falla en cuanto intento de elucidación filosófica del concepto de significado" y, además, que el enfoque de McDowell “... no puede ser considerado, tal y como

34 Publicado en Teorema.

35 Cf. J. McDowell (1976), (1977). 
se ha pretendido, un perfeccionamiento de la teoría del significado propuesta por el propio Davidson". ${ }^{86}$

Hasta donde llega mi conocimiento de la obra de McDowell, en ningún lugar se dice que la propuesta a defender debe entenderse como "un perfeccionamiento" de la teoría davidsoniana del significado. Es cierto que GS-VV también discuten la defensa de la teoría de McDowell que Mark Platts ha intentado llevar a cabo en su libro Ways of Meaning ${ }^{37}$ $y$, probablemente, Platts ha entendido a la obra de McDowell como "un perfeccionamiento" de la teoría de Davidson, o, al menos, como un desarrollo de la misma. Pero no así el autor de la teoría. Claro está que el trabajo semántico de quienes comparten el supuesto aludido en el párrafo anterior, estará por siempre en deuda con la obra de Davidson. Es a Davidson a quien debemos la idea de utilizar una definición recursiva de la verdad para explicar el significado de las oraciones de un lenguaje. $Y$ cualquier filósofo interesado en discutir seriamente la cuestión de una teoría del significado deberá comenzar por reconocer este hecho.

Naturalmente, John McDowell no es una excepción. Pero su teoría difiere, radicalmente, de la teoría de Davidson: en la teoría de McDowell no encontramos, ni explícita ni implícitamente, alusión alguna a la teoría de la decisión. Tampoco se reconocen los méritos -o la necesidadde la noción davidsoniana de considerar a una oración como verdadera, tan crucial en la teoría de Davidson de la interpretación radical. Esto separa de manera fundamental los caminos que han de seguir ambas teorias, pero, obviamente, esto no significa que no compartan algunos supuestos igualmente fundamentales. Lo que interesa preguntarse es, entonces, cuáles son las razones por las que McDowell no se anima a examinar, como lo hace Davidson, la estrecha conexión que existe entre una teoría de la interpretación, o del significado, y la teoría de la decisión. En otras palabras, ¿qué es lo que reemplaza, en la teoría de McDowell, a la teoría de la decisión? ¿Por qué, en la teoría de McDowell, no hace falta una noción análoga a la de asentimiento en la filosofía de Quine - noción la cual tiene como correlato, en la teoría de Davidson, la de considerar a una oración como verdadera (holding true)?

Antes de intentar contestar estas preguntas, repasemos los supuestos compartidos por ambas teorías: uno de ellos es precisamente el requisito de que una teoría del significado sea interpretativa, es decir, que sea de una naturaleza tal que su conocimiento sea suficiente para entender el lenguaje de que se trata. Otro supuesto en común sería el que una teoría tal tenga, como uno de sus componentes, una teoria de la verdad del tipo de la de Tarski, para el lenguaje en cuestión. Ahora bien, McDowell se

38 Cf. "Significado, verdad y comprensión", p. 27.

37 Cf. M. Platts (1979). 
interesa por enunciar este último requisito en un contexto fregeano. Nos dice, por ejemplo

...Frege sostuvo que los sentidos de las oraciones pueden determinarse mediante la adscripción de condiciones de verdad, y que el sentido de los componentes oracionales es su contribución al sentido de las oraciones en las que figuran. El paralelo [con la teoría de Davidson] es sorprendente. Sugiere interpretar la propuesta davidsoniana como una propuesta acerca de la naturaleza de una teoría (fregeana) del sentido para un lenguaje. ${ }^{38}$

Hemos visto ya cómo, desde la publicación del artículo seminal "Truth and Meaning" Davidson estaba consciente de que no toda teoría que asignase, correctamente, condiciones de verdad a las oraciones de un lenguaje, podría ser considerada como una teoría de la interpretación. McDowell, como es natural, piensa lo mismo. Pero, la terminologia que usa sigue los pasos de Frege:

... no... cualquier teoría de la verdad, por verdadera que sea, podría servir como una teoría del sentido. ${ }^{39}$

¿Qué debemos agregar, según McDowell, a la teoría de la verdad para que pueda ésta figurar o servir como una teoría del sentido?

Según McDowell, un teoría que asigne condiciones de verdad debe interactuar con una teoria de la fuerza, la cual tiene como objeto dos cosas: por una parte, permitirnos identificar acciones lingüísticas qua actos proposicionales de ciertos tipos, o sea, aserción, preguntas, órdenes, etc., y, por la otra, mostrarnos cómo extraer, a partir de una descripción suficientemente detallada de las emisiones de un hablante, una oración indicativa $o$ del lenguaje objeto. McDowell resume así su posición:

La idea es que una teoría del sentido y una teoría de la fuerza, en forma combinada, nos permitan pasar, de una descripción suficientemente detallada de las emisiones de un hablante no interpretadas, a una descripción de su emisión como un acto proposicional de un tipo especifico y con un contenido especifico, esto es, una descripción de la forma "El está diciendo que p", "El está preguntando si $\mathrm{p}$ ", etc.; en donde lo que reemplaza a 'p' . . es la oración usada en el lado derecho del teorema que la teoría del sentido implica para cada oración indicativa la cual es certificada por la teoría de la

s8 Cf. J. McDowell (1976), p. 141 de la antología de Platts, cf. M. Platts (1980), p. 5. de la traducción.

38 lbid., p. 6 de la traducción. 
fuerza como relacionada en la forma adecuada con la emisión en cuestión...40

Ahora bien, una teoría bipartita del significado, como la que interesa a McDowell, debe verse como parte de una teoría global de la conducta. Se trata de redescribir lo que en un principio apareciera como emisiones estructuradas de sonido, como actos de habla de tipos específicos con contenidos específicos. Es aquí en donde sentimos el peso de la noción de sentido, de esa noción cuyo interés estriba en ayudarnos a entender la conducta de las personas y, en última instancia, a las personas mismas. Necesitamos, pues, verificar si los resultados que arroja la teoría bipartita nos permiten adscribir actitudes proposicionales, o sea, creencias y deseos, inteligibles a la luz de las creencias y demás actitudes proposicionales del intérprete mismo y de las observaciones que ha llevado a cabo de las acciones de los nativos, de sus emisiones lingüísticas y del contexto de estas últimas. Así, podría decirse que la teoría del sentido requiere tan solo ver en sus teoremas meros apareos de designaciones de oraciones del lenguaje objeto con oraciones del metalenguaje: formas esquemáticas del tipo

$$
s \ldots p
$$

La idea es que una vez que se ha tomado en cuenta el requisito adicional de que la teoría bipartita nos permita dar un contenido inteligible a las actitudes proposicionales de los hablantes, podemos llenar el espacio en blanco introduciendo el predicado "...es verdad" y el bicondicional correspondiente. Pero es el hecho de que las oraciones que ocupan el lugar de ' $\mathrm{p}$ ' puedan utilizarse para fijar el contenido de los actos de aserción, etc., a que se de lugar mediante la emisión de oraciones indicativas, es este hecho, repito, lo que garantiza que una teoría de la verdad pueda a la vez calificar como una teoría de la interpretación, esto es, como una auténtica teoría del sentido. Así, McDowell escribe:

El hecho de que las oraciones usadas especifiquen el contenido de los dichos potencialmente efectuados mediante la emisión de las oraciones mencionadas, garantizaría que el predicado que pudiera escribirse, si quisiéramos, fuese "verdadero"; garantizaría que la teoría, con sus teoremas escritos de esa manera, fuese una teoría verdadera de la verdad. ${ }^{41}$

Pero, como dice McDowell a continuación:

40 Cf. J. McDowell (1976), p. 44.

41 Cf. J. McDowell (1977), p. 7 de la traducción. 
...sería el hecho que garantiza, y no el hecho garantizado, lo que adecuó a la teoría para servir como una teoría del sentido. ${ }^{42}$

Si bien es cierto que el hecho que garantiza que una teoría de la verdad califique como una teoría de la interpretación es, precisamente, el exigir que las oraciones contenido -o sea las oraciones que ocupan el lado derecho de los bicondicionales de las oraciones-V - sirvan para adscribir un contenido específico a las actitudes proposicionales de los hablantes, es de suma importancia notar que esto no significa que se haya otorgado una prioridad conceptual a las nociones del círculo intensional -creencias, deseos, intenciones, etc.-, como sucede en la teoría del significado y de la comunicación desarrollada por P. Grice.43 Fijar el contenido de las actitudes proposicionales de los hablantes debe también estar ceñido a las consideraciones estructurales que derivan de la naturaleza misma de la teoría del sentido. En otras palabras, la teoría del sentido nos obliga a ver el contenido de una aserción que el hablante lleva a cabo mediante la emisión de una oración, como el resultado de la contribución semántica de los componentes oracionales a todas las oraciones en las que figuran, particularmente, la oración que el hablante usó para expresar una creencia. Se trata, pues, de construir una teoría del significado y una teoría de las actitudes proposicionales mostrando cómo los elementos de cada una de éstas interactúan entre sí; el afán no es reducir unos conceptos, v. gr., los conceptos semánticos, a otros, i.e., las nociones psicológicas como la creencia, el deseo y la intención: "Lo que proponemos aqur" nos dice McDowell

no es elucidar la noción de sentido en base a otras nociones, y mucho menos reducirla a aquellas otras; sencillamente nos proponemos una descripción de su relación con esas otras nociones: la esperanza es que una noción, a primera vista problemática, pueda hacerse menos problemática al mostrarse su lugar, por asi decirlo, en un espacio conceptual en el que normalmente nos las arreglamos sin necesidad de pensar. ${ }^{44}$

Tenemos, pues, dos requisitos - uno externo y el otro interno- que debe satisfacer toda asignación de contenido a las actitudes proposicionales: el requisito externo nos exige que dichas actitudes proposicionales resulten inteligibles y el requisito interno nos obliga a ver el contenido de las actitudes proposicionales expresadas mediante la emisión de ciertas oraciones como una función de la contribución repetible de sus

42 Ibid.

43 P. Grice (1957).

4 J. McDowell (1976). 
componentes. Nótese que ni los argumentos de McDowell, ni aquellos propuestos por el profesor Davidson, presuponen que nuestro punto de partida sea la doctrina, lisa y llana, de que el significado de una oración nos lo dan sus condiciones de verdad. Ambos autores comienzan por hacer consideraciones de un tipo mucho más general: McDowell nos dice que una teoría del significado ha de verse como parte de la teoría global que pretende hacernos inteligible la conducta, lingüística y de otros tipos, de los hablantes de un lenguaje; Davidson alude constantemente al propósito o el interés de una teoría del significado, insistiendo enfáticamente en que el problema del significado debe ubicarse dentro de un contexto muchísimo más amplio, a saber, el contexto de las actitudes proposicionales y de la acción intencional como ésta se muestra en el proceso de la interpretación radical. Cuando se han apreciado estas consideraciones, caemos en la cuenta que la teoría que tenemos ante nosotros es una teoría de la verdad. Como Platts escribe: "...las reflexiones generales acerca del papel de una teoria del significado dentro de una explicación de la conducta puede apreciarse antes de cobrar conciencia de lo adecuado del predicado veritativo".45 En realidad, el propósito ha sido, como Wiggins nos dice en su reciente contribución al Festschrift de Strawson, aligerar el requisito de traducción impuesto por Tarski mediante la idea davidsoniana de la interpretación radical y las nociones generales de explicación, comprensión y entendimiento. ${ }^{46}$ Dichas consideraciones, como ya hemos visto, hacen posible discutir las restricciones que han de imponerse a una teoria que aparee nombres de oraciones del lenguaje objeto y oraciones del metalenguaje

$$
s \ldots p
$$

sin todavía saber cuál es exactamente el predicado que habrá de llenar el espacio en blanco. Satisfechas las restricciones en cuestión, sabemos que ' $p$ ' puede utilizarse para determinar el contenido de los actos proposicionales que realicen los hablantes y si esto es así, tenemos ya la garantía de que el espacio en blanco podrá llenarse con el predicado “... es verdad" y el bicondicional correspondiente, obteniendo así:

(V) $S$ es $V$ sii $p$.

Pero, si lo que acabo de decir está en lo correcto, entonces, el paso de " $s . . p$ " a "s es $\emptyset$ sii $p$ " puede darse con la garantía de que ' $p$ ' será en verdad una oración que nos dé el significado o el contenido de los actos

45 Cf. M. Platts (1979), p. 61.

to Cf. D. Wiggins (1980). 
proposicionales independientemente del predicado que sea $\emptyset$, el predicado "... es $V$ " o quizás algún otro. En el artículo de Wiggins que acabo de citar, encontramos lo siguiente:

Al final hay tantas restricciones colaterales diversas sobre cualquier [teoría del sentido] $\Theta$ que nos indique el algoritmo para anexar, a una oración arbitraria, un contenido adecuado para propósitos de interpretar las emisiones de $o$, que ni siquiera tenemos que estipular que $\Theta$ sea una teoría de la verdad.47

Pero la idea de McDowell es que, aunque no tenemos que considerar a $\Theta$ como una teoría de la verdad, podemos hacerlo. Si llenásemos la laguna entre ' $s$ ' y ' $p$ ' mediante otro predicado que no fuese "....es verdad", de todas maneras sabríamos que una teoría del sentido especificaría condiciones de verdad: directamente o via una conversión justificada. ${ }^{48}$

La idea central del programa de McDowell estriba en vincular, tan estrechamente que resulte imposible divorciarlos, el contenido de los actos proposicionales, fundamentalmente, de la aserción, y las asignaciones de contenido que obtenemos como resultado de una teoría del sentido. McDowell se refiere a este vínculo como el requisito de interacción entre una teoría del sentido y una teoría de la fuerza. En su artículo "Truth Conditions, Bivalence and Verificationism", nos dice:

La aserción no es un fenómeno casualmente observable que podemos describir, ahí donde ocurre, independientemente de la construcción de una teoría sistemática para el lenguaje en el que se dé.99

Volvamos por un momento al artículo de GS-VV. Ambos reconocen la importancia del requisito de interacción en la teoría de McDowell. Empero, critican la teoría sobre la base de que el output de una teoría de la fuerza sea tan sólo la conclusión de que el hablante asevera (ordena, etc.), algo, sin tener la más remota idea de lo que sea esto. $Y$ aunque reconocen que "Ciertamente, la teoría de la fuerza tiene otro componente que suministra, para cada emisión una oración indicativa" tienen razón al afirmar que "... este componente no es, por cierto, interpretativo". Lo que sencillamente constituye un non-sequitur es su conclusión de que "El trabajo de interpretación corre de cuenta de la teoría del sentido por vía de las oraciones contenido usadas en el lado derecho de los teoremas". Dicha conclusión pasa por alto que lo que está en

47 Tbid., p. 200.

48 Cf. J. McDowell (1976), p. 47.

49 En la p. 52. 
juego es un requisito de interacción entre la teoría de la fuerza y la teoría del sentido que debe cumplir cualquier teoría del significado, cualquier teoría que nos explique el funcionamiento de un lenguaje. Sería tan falso afirmar, como lo hacen GS-VV, que "el trabajo de interpretación corre por cuenta de la teoría del sentido" como afirmar que dicha labor corre por cuenta de la teorfa de la fuerza. Ninguna de estas dos teorías, por sí mismas, realizan esta función. Por eso se habla de una teoria bipartita del significado.

\section{BIBLIOGRAFIA}

Davidson, D. (1967), "Truth and Meaning", Synthese, 17.

- (1970), "Semantics for Natural Languages", en Linguaggi nella Societá e nella Tecnica (Edizione di Comunitá, Milano).

- (1973 a), "Radical Interpretation", Dialectica, 27.

(1973 b), "In Defense of Convention T", en Leblanc, H. (ed.), Truth, Syntax and Modality (North-Holland).

- (1974), "Belief and the Basis of Meaning", Synthese, 27.

- (1976), "Reply to Foster", en Evans, G. y McDowell, J. (eds.), Truth and Meaning (Oxford University Press).

(1979), "Moods and Performances", en Margalit, A. (ed.), Meaning and Use (Reidel).

- (en prensa), "Towards a Unified Theory of Meaning and Action".

Poster J. (1976), "Meaning and Truth Theory" en Evans, G. y McDowell, J. Truth and Meaning, op. cit.

Grice, P. (1957), "Meaning", Philosophical Review, 66.

García Suárez, A. y Valdés Villanueva, L. (1982), "Significado, verdad y comprensión" en Teorema.

McDowell, J. (1976), "Truth Conditions, Bivalence and Verificationism", en Evans, G. y McDowell, J., Truth and Meaning, op. cit.

- (1977), "On the Sense and Reference of a Proper Name", Mind, 86. (Hay traducción al español de Lourdes Valdivia en Cuadernos de Crítica, Nọ 20, UNAM, 1981.

Platts, M. (1979), Ways of Meaning (Routledge and Kegan Paul).

- (1980), (ed.), Reference, Truth and Reality (Routledge and Kegan Paul). Tarski, A. (1956), "The Concept of Truth in Formalized Languages", en Logic, Semantics, Metamathematics (Oxford University Press).

Wiggins, D. ((1980), "What would be a substantial theory of truth?" en van Straaten, Z. (ed.), Philosophical Subjects (Oxford University Press). 